\title{
SOPORTE TÉCNICO DE SIMULACIÓN PHET EN LA ENSEÑANZAY APRENDIZAJE DE FRACCIONES EQUIVALENTES
}

Jorge Enrique Díaz Pinzón*

*Docente titular de Matemáticas I.E. General Santander. E-mail: jediazp@unal.edu.co, jorgediaz333@gmail.com.

Recibido: 20 Junio de 2016

Aceptado: 12 Septiembre de 2016

*Correspondencia del autor: Jorge Enrique Díaz Pinzón. E-mail: jorgediaz333@gmail.com

\section{RESUMEN}

Este trabajo de investigación tuvo por objetivo comparar el resultado de cada estudiante con respecto a cada nivel de dificultad con la aplicación de Simulaciones Phet, en la enseñanza y el aprendizaje de fracciones equivalentes, fracciones mixtas y reducción de fracciones. Esta Investigación es de tipo experimental - cuantitativa, con un único grupo (Prueba con el simulador Phet). La población de estudio estuvo constituido por 40 estudiantes de octavo grado de educación secundaria de la Institución Educativa General Santander de Soacha-Cundinamarca. El grupo de estudio estuvo conformado por 40 estudiantes ( 20 hombres y 20 mujeres). Se realizó una comparación múltiple entre medias independientes, a partir del análisis estadístico de los datos aplicando la prueba Scheffé, se pudo observar que hay evidencia para rechazar la hipótesis nula, por tanto las medias son significativamente diferentes. La nota promedio fue 9,575 para el primer nivel, de 9,710 para el segundo nivel, de 9,385 para el tercer nivel, 8,840 para el cuarto nivel, 8,370 para el quinto nivel, 7,610 para el sexto nivel, 8,478 para el séptimo nivel, 7,530 para el octavo nivel. La aplicación del simulador Phet permitió un aprendizaje activo, participativo, característico, acrecentando el nivel de participación del estudiante, cumplimiento de tareas y actividades, y el interés por el tema de estudio, lo cual quedó demostrado.

Palabras claves: Técnica de Simulación, Tecnologías de la Información, Estrategia educativa, Técnica didáctica. Inferencia estadística. 


\begin{abstract}
This research aimed to compare the result of each student for each level of difficulty with the application of simulations Phet, teaching and learning equivalent fractions, mixed fractions and reduction of fractions. This research is experimental - quantitative, with one group (Try the simulator Phet). The study population consisted of 40 eighth graders of secondary education of School General Santander de Soacha-Cundinamarca. The study group consisted of 40 students ( 20 men and 20 women). a multiple comparison of independent means, from the statistical analysis of data using the Scheffe test was performed it was observed that there is evidence to reject the null hypothesis, so the means are significantly different. The average was 9,575 for the first level of 9,710 for the second level of 9,385 for the third level, 8,840 for the fourth level, 8,370 for the fifth level, 7,610 for sixth level, 8,478 for the seventh level, 7,530 for the eighth level. The application of the simulator Phet allowed an active, participatory learning characteristic, increasing the level of student participation, fulfillment of tasks and activities, and interest in the subject of study, which was demonstrated.
\end{abstract}

Keywords: Simulation Technique, Information Technology, Educational Strategy, teaching technique. Statistical inference.

\section{INTRODUCCIÓN}

La Internet es considerada en la actualidad como uno de los más importantes fenómenos del comienzo del siglo XXI. Su desarrollo, unido al extraordinario progreso en todas las técnicas de comunicación e información, puede ser comparado con el nacimiento de la escritura o de la imprenta.

El desarrollo de las nuevas tecnologías de la información y la comunicación relacionadas con la Internet, ha abierto nuevos espacios en el ámbito educativo. El diseño de sitios Web que integran múltiples aplicaciones multimedia, está dotando a los docentes en general, de nuevos ambientes de aprendizaje donde predomina la interactividad y el rol del profesor innovador, facilitando al estudiante la construcción de su propio conocimiento.

No obstante, se debe tener en claro que si bien la tecnología educativa es un elemento importante para mejorar los procesos de enseñanza - aprendizaje, esta mejora no depende solamente de la utilización de un software educativo, sino de su adecuada integración curricular, es decir, del entorno educativo diseñado por el docente.

La problemática radica en que el estudiante se le dificulta representar situaciones diversas que involucren los diferentes usos de las fracciones en base a disímiles contextos, por tal motivo el diseño y adaptación de simulación de la plataforma PhET permite el estudio de las fracciones equivalentes en términos de encontrar fracciones equivalentes usando números y gráficas, enlazar o emparejar fracciones con diferentes tipos de gráficas, esto contribuye y facilita el aprendizaje del concepto de fracciones equivalentes.

Mediante el uso de Simulaciones Phet como una ilustración animada, docentes, encontramos que es más fácil comunicarse efectivamente con sus estudiantes. Los Phet muestran procesos dinámicos y estos pueden ser lentos abajo, acelerado, o en pausa, dependiendo del concepto que se muestra; lo invisible se hace visible; y múltiples representaciones están vinculados. Por último, los sims se ajustan fácilmente por el instructor durante el debate. Estas características hacen a menudo sims más eficaces para el aprendizaje y más práctico utilizar los dibujos estáticos o demostraciones en vivo.

Phet está diseñado para ayudar a los estudiantes a desarrollar habilidades de investigación científica mediante la exploración de las relaciones de causa y efecto.

Los instructores pueden facilitar la consulta de toda la clase mediante la creación de un escenario en la simulación, y pidiendo a los estudiantes que predigan el efecto de variables manipulando. En estos salones de clase, los estudiantes a menudo de forma espontánea preguntan muchos más, y más profundo preguntas. 
Es común que los estudiantes piden una serie de "qué pasaría si" preguntas y dirigir el uso de la tarjeta SIM de los profesores.

Simulaciones Phet permiten tareas utilizar un enfoque de investigación guiada donde los estudiantes participar en la exploración-científico como, un enfoque que normalmente podría requerir un instructor presente para facilitar el progreso de los estudiantes.

Ahora bien el propósito desde objeto virtual de aprendizaje (OVA), es que los estudiantes sean capaces de: Encontrar fracciones usando números y las imágenes que coincidan, hacer las mismas fracciones usando disímiles números, fracciones en diferentes patrones de imagen, comparar fracciones usando números $\mathrm{y}$ patrones de fondo.

Este objeto de aprendizaje puede utilizarse con estudiantes de diferente edad y nivel de grado que necesitan ayuda con fracciones. La simulación se mezcla múltiples representaciones de fracciones de 0-6 en ambas formas impropias y mixtas. Los estudiantes no tienen que manejar formas reducidas de las fracciones, sino que más bien se relacionan muchas representaciones como $1 / 2=4 / 8$ e imágenes de objetos como pasteles y plazas en rodajas en pedazos (Loeblin, 2016).

¿Cómo funciona esto? La simulación está diseñado para dar señales a los estudiantes a explorar de manera productiva mediante el uso de implícita (en contraposición a explícito) guía - con la elección de los controles, las representaciones visuales, y la retroalimentación inmediata proporcionada por visual cambios como los estudiantes a explorar. Este enfoque permite que las tareas sean menos dirigió y utilizar más conceptual abierta preguntas. (Muñoz, 2012,p.1)

Ahora los simuladores: "Son objetos de aprendizaje que mediante un programa de software, intentan modelar parte de una réplica de los fenómenos de la realidad y su propósito es que el usuario construya conocimiento a partir del trabajo exploratorio, la inferencia y el aprendizaje por descubrimiento". (Peña \& Alemán, 2013,p.1)

También según (Fatela, 2012,p.1) "Los simuladores digitales son aplicaciones interactivas que simulan situaciones de experimentos físicos reales o que ilustran temas matemáticos".

Rev. Invest. Univ. Quindío (Col.), 28(2): 31-41; 2016
Los simuladores son programas que representan un modelo o entorno dinámico y en que a través de gráficos o animaciones facilitan al estudiante la visión de lo que ocurre en el entorno que se está simulando, de forma que modificando de manera interactia las características del entorno puede comprender mejor lo que sucede en el entorno que está intentando conocer. Dada la actualización de la tecnología, siempre debemos estar en busca de nuevos simuladores que sean más efectivos e interesantes.

(Ortega, 2001,p.276).

Esto en consonancia con (Bagur, 2011, p.2) "En lo personal, creo que para que estos simuladores tengan todo el éxito que pueden lograr es necesario, primero, que el docente identifique muy bien los elementos del tema a enseñar; que trate el tema por medio de algunos ejemplos y luego use a los simuladores como medios de práctica o evaluación".

Los simuladores matemáticos ofrecen variedades de temas en esta área del conocimiento, contienen una explicación muy didáctica, divertida, entretenida y sobre todo con la mayor claridad posible, con muchos ejemplos de aplicación a la vida cotidiana para que el usuario le saque el mejor provecho a este tipo de herramientas que abundan en internet. (Duran, 2012,p.1).

De acuerdo con (Muñoz, 2012, p.1) "Es una página de gran valor didáctico con muy entretenidas e interactivas simulaciones gratuitas apoyados en investigaciones del proyecto PhET de la Universidad de Colorado".

Dado la importancia de la utilización de Simuladores como herramienta aprendizaje varios autores mencionan lo siguiente: Galicia (2005) en su tesis las aulas virtuales en el proceso enseñanza aprendizaje en el nivel medio, ciclo básico, sector privado del área urbana del municipio de Jalapa de la Facultad de Humanidades de la Universidad de San Carlos de Guatemala, destaca el progreso de la ciencia y la tecnología y el uso del computador como una herramienta necesaria en todos los ámbitos sociales.

Añade (Arbeláez,2010) en su tesis mundos virtuales para la educación en salud simulación y aprendizaje en Open Simulator, en la universidad de Caldas, Manizales, Colombia, que el uso de simulación y de videojuegos se ha transferido con fuerza en la industria, prácticas militares y medicina, generando en los últimos años en una poderosa herramienta de formación, en lo relacionado a la medicina se han desarrollado simuladores de equipos y dispositivos de proce- 
dimientos de alto riesgo y simuladores para fortificar procesos educativos en la salud, estos instrucciones se han introducido a otras áreas como lo es la educación.

De igual forma (Pósito, 2012) menciona que los modernos avances tecnológicos actuales han logrado grandes impactos en la educación, ampliando los escenarios educativos, brindando medios de comunicación y soporte de materiales para proporcionar la interacción entre las personas. Se planteó el objetivo de ofrecer soluciones tecnológicas y pedagógicas al problema del diseño de habilidades de aprendizaje para aprender Ciencias Naturales en los nuevos contextos educativos.

Contribuye (García, 2012) en la tesis titulada promover en el aula estrategias de aprendizaje para elevar el nivel escolar de los estudiantes de tercero primaria en el área de Matemática de la Facultad de Humanidades de la Universidad Rafael Landívar, Guatemala, al cavilar sobre las insuficiencias en el aprendizaje de las Matemáticas por diferentes factores y despunta la importancia de utilizar estrategias de aprendizaje para perfeccionar el nivel académico del estudiante.

Como norma general se puede decir que convendrá utilizar un determinado programa cuando su empleo aporte más ventajas que la aplicación de otros medios didácticos alternativos. Y en cuanto a la forma de utilización, nuevamente será la que proporcione más ventajas. (Márquez,

\section{Metodología}

El proyecto se realizó mediante un tipo de investigación experimental, este tipo de investigación es:

Aquella que permite como mayor seguridad establecer relaciones de causa a efecto". Pues presenta una visión general y aproximada del objeto de estudio, además de contar una investigación de tipo cuantitativo. Se ha escogido una metodología de tipo cuantitativa, el diseño de la investigación cuantitativa establece un método experimental habitual del conjunto de las normas científicas. (Monje, 2011,p.105).

Regularmente, a estos experimentos se los nombra ciencia verdadera y manejan medios matemáticos y estadísticos cotidianos para evaluar los resultados de modo concluyente.
Todos los experimentos cuantitativos utilizan un formato estándar, con algunas pequeñas diferencias inter-disciplinarias para generar una hipótesis que será probada o desmentida. Esta hipótesis debe ser demostrable por medios matemáticos y estadísticos y constituye la base alrededor de la cual se diseña todo el experimento. (Shuttleworth, 2008,p.1).

Este tipo de metodología, permite al investigador diseñar ambientes de aprendizaje, lo cual implica una mayor comprensión de sus elementos (estudiante, objeto matemático, contexto, artefacto o recurso tecnológico, situación-problema, actividad estructurada, etc.), así como anticipar como estos van a funcionar conjuntamente para promover el aprendizaje. Según Cobb et al. (2003), citado

por (Huapaya,2012,p.56)

Una de las particularidades de esta metodología es que nos permite asumir el rol de investigadores y docentes, de esta manera se podrá variar y tomar decisiones en los experimentos prescritos. La segunda característica es de intervención, pues proponemos mejorar el aprendizaje de fracciones equivalentes, fracciones mixtas y reducción de fracciones a través de prácticas de simulación de situaciones-problema mediadas por el Phet.

La estrategia didáctica que se empleó en la experiencia con el uso de simulación Phet. En función de identificar fracciones equivalentes, mixtas y reducción de fracciones. La estrategia implicó dos etapas:

Instruccional: Para preparar y motivar al estudiante en qué y cómo iba a aprender. En ésta fase se explicó en qué consistía la experiencia, indicándose además el sistema de evaluación, actividades a desarrollar en el computador, manejo del software de simulaciones (Phet).

Postinstruccional: Para fomentar el desarrollo de un aprendizaje significativo, se introdujo el contenido temático de fracciones equivalentes, fracciones mixtas y reducción de fracciones. Se desarrollaron ejercicios, en un principio con ejemplos visualizados a través del software de simulaciones (Phet) Figura 1. Y luego el estudiante desarrolló los ejercicios en cada uno de los ocho niveles para certificar los resultados a través del mismo programa. 


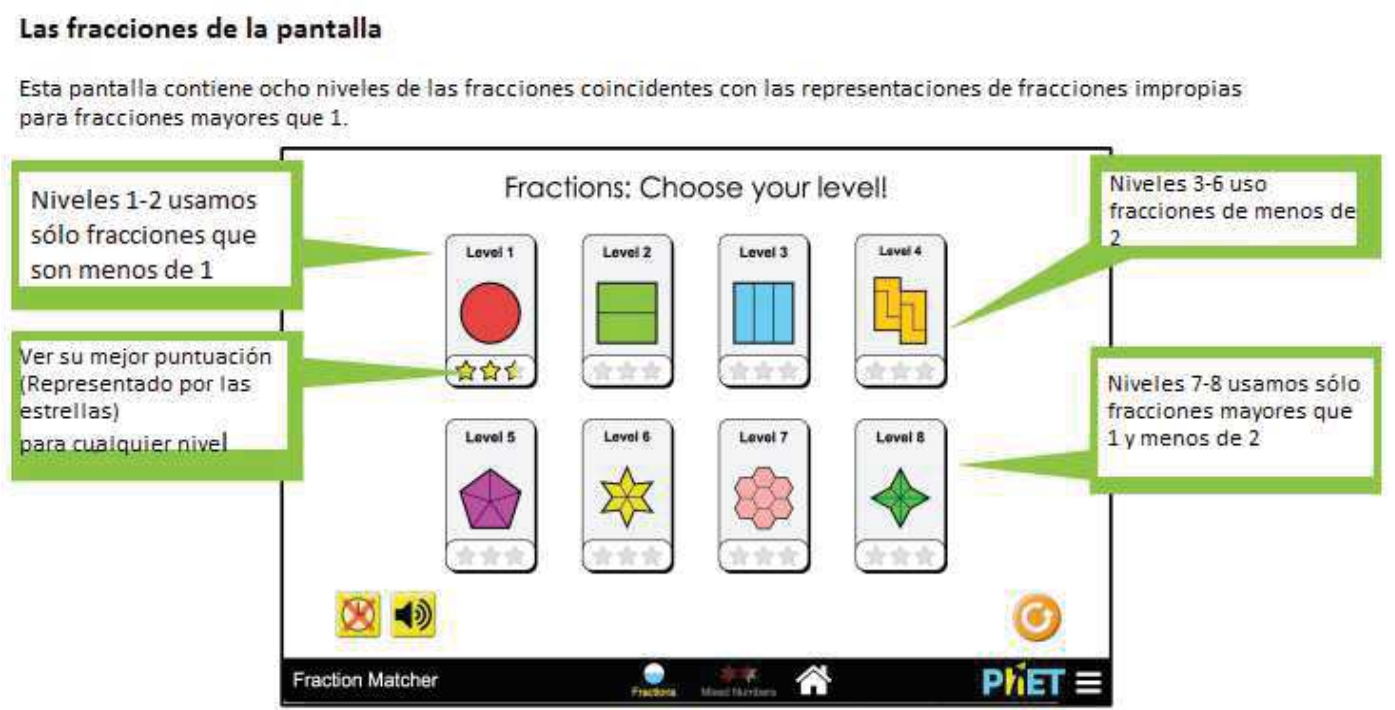

Figura 1. Niveles del simulador Phet. Fuente: (McGarry, 2015)

\section{Población}

La población según (Balestrini,2006,p.126), se define como "cualquier conjunto de elementos de la que se quiere conocer o investigar alguna de sus características". Este proyecto se centra en estudiantes de la jornada de la tarde, específicamente con estudiantes grado octavo cuyas edades oscilan entre los 12 y 15 años.

\section{Muestra}

La muestra está representada por los estudiantes del grado octavo.

Fórmula para calcular el tamaño de la muestra

Se utilizó la siguiente fórmula para calcular el tamaño de la muestra:

$n_{e}=\frac{Z^{2} v^{2} N}{e^{2}(N-1)+Z^{2} v^{2}}$

Dónde:

$\mathrm{n}=$ el tamaño de la muestra.

$\mathrm{N}=$ tamaño de la población.

$v=$ Desviación estándar de la población que, generalmente cuando no se tiene su valor, suele utilizarse un valor constante de 0,5 .

$\mathrm{Z}=$ Valor obtenido mediante niveles de confianza. Es un valor constante que, si no se tiene su valor, se lo toma en relación al 95\% de confianza equivale a 1,96 (como más usual) o en relación al 99\% de confianza equivale 2,58 , valor que queda a criterio del investigador.

e = Límite aceptable de error de la muestra que, generalmente cuando no se tiene su valor, suele utilizarse un valor que varía entre el $1 \%(0,01)$ y $9 \%(0,09)$, valor que queda a criterio del encuestador.

Se tiene $N=43$, para el $99 \%$ de confianza $Z=2,58$, y como no se tiene los demás valores se tomará $\delta=0.5 \mathrm{y}$ $\mathrm{e}=0,05$.

Reemplazando los valores en la fórmula se obtiene:

$$
\begin{aligned}
& n_{e}=\frac{N v^{2} Z^{2}}{(N-1) e^{2}+v^{2} Z^{2}} \\
& \mathrm{n}_{e}=\frac{43 \$ 0,5^{2} \$ 2,58^{2}}{(43-1)(! 0,05)^{2}+0,5^{2} \$ 2,58^{2}}=\frac{71.55}{1.76}=40.4=40
\end{aligned}
$$

\section{Resultados}

En la figura 1, se observan los promedios obtenidos por cada uno de los estudiantes en forma ascendente, que van desde 5,625 hasta 10,0 se puede observar la gran variedad de promedios en los diferentes niveles realizados por los cuarenta estudiantes. En la figura 2 se aprecian las medias de calificación en cada nivel de complejidad del simulador Phet. 


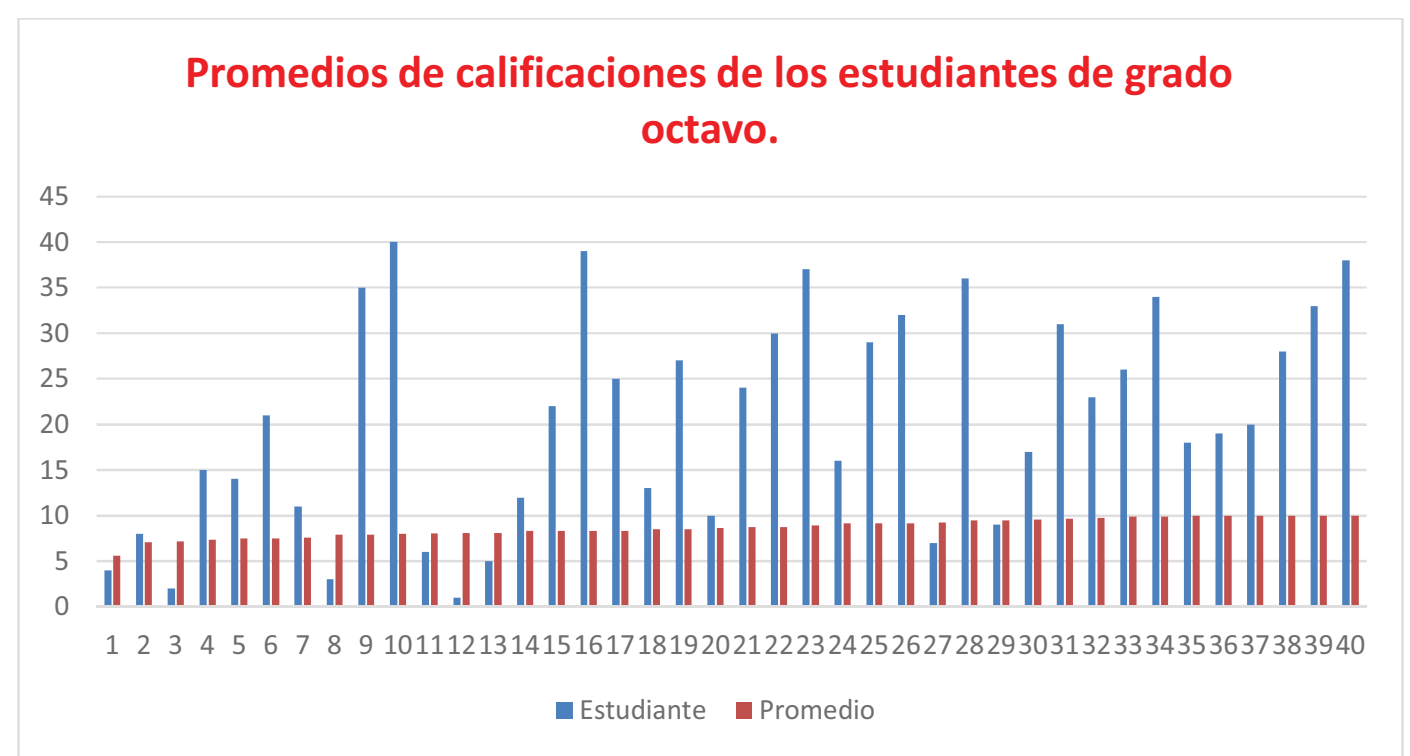

Figura 1.Promedios de calificaciones de los estudiantes de grado octavo. Fuente: Autor

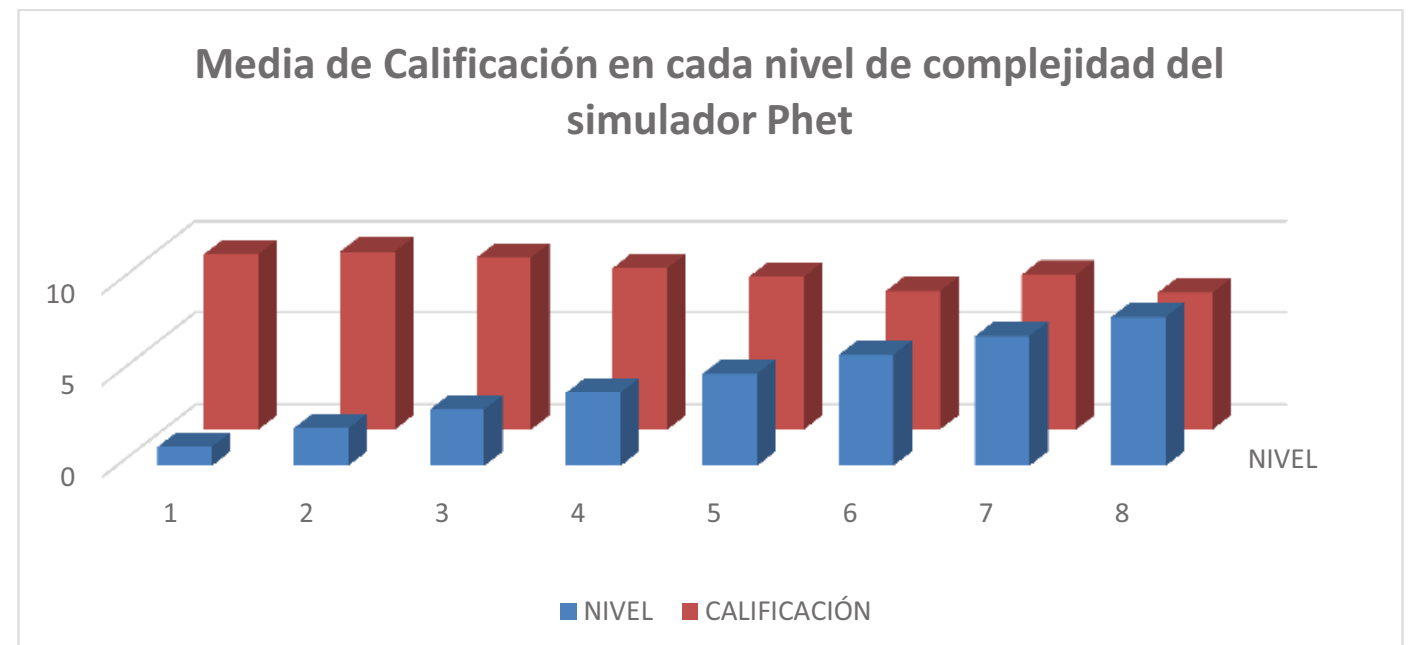

Figura 2. Media de calificación en cada nivel de complejidad del simulador Phet. Fuente: Autor

\section{Hipótesis con el ANOVA}

La hipótesis del presente trabajo de investigación se diseña como una relación causal y se enuncia de la siguiente forma:

Hipótesis Alterna (Ha): Con la aplicación del uso del Simulaciones Phet, para al menos uno de los niveles del simulador, en el promedio de calificación hay diferencias en el promedio de puntajes de los estudiantes en cada nivel, en el tema de fracciones en los estudiantes del grado octavo.

Hipótesis Nula (Ho): Con la aplicación del uso del Simulador Phet, No hay diferencias en promedios de puntajes de los estudiantes en cada nivel, en el tema de fracciones en los estudiantes del grado octavo.

Prueba Estadística: El valor de significancia de la prueba es de $\alpha=0.05$ (5\%), si es mayor se acepta la hipótesis nula si es menor se rechaza la hipótesis nula. Comparación múltiple entre medias independientes, se aplicó la prueba de Scheffé, para diferencia de medias en muestras independientes. Se utilizará el Software SPSS v 23.0.

En la tabla 1, se observa que el p-valor de la prueba es igual a 0,000 es menor a $\alpha=0.05$, de esta manera se rechaza Ho, es decir que para al menos uno de los promedios es diferente de los demás. 
Tabla 1. ANOVA

\begin{tabular}{llccccc}
\hline & & $\begin{array}{c}\text { Suma de } \\
\text { cuadrados }\end{array}$ & gl & $\begin{array}{c}\text { Media } \\
\text { cuadrática }\end{array}$ & F & Sig. \\
\hline \multirow{2}{*}{ CALIFICACIÓN } & Entre grupos & 335,899 & 39 & 8,613 & 3,901 &, 000 \\
& Dentro de grupos & 618,219 & 280 & 2,208 & & \\
& Total & 954,117 & 319 & & & \\
\hline Fuente: Autor & & & &
\end{tabular}

Ahora cuando Ho se rechaza en la prueba ANOVA, entonces se procede a realizar una prueba Post Hoc, en este caso la prueba de Scheffe para muestras independientes y observar si hay diferencias entre cada uno de los niveles, como se puede observar en la tabla 2.

Tabla 2. Prueba de Scheffe-Comparaciones múltiples

\begin{tabular}{|c|c|c|c|c|c|c|}
\hline \multirow[t]{2}{*}{ NIVEL } & & \multirow{2}{*}{$\begin{array}{l}\text { Diferencia de } \\
\text { medias (I-J) }\end{array}$} & \multirow[t]{2}{*}{ Error estándar } & \multirow[t]{2}{*}{ Sig. } & \multicolumn{2}{|c|}{ Intervalo de confianza al $\mathbf{9 5 \%}$} \\
\hline & & & & & Límite inferior & Límite superior \\
\hline \multirow{8}{*}{1} & 2 & $-0,135$ & 0,3477 & 1 & $-1,449$ & 1,179 \\
\hline & 3 & 0,19 & 0,3477 & 1 & $-1,124$ & 1,504 \\
\hline & 4 & 0,735 & 0,3477 & 0,724 & $-0,579$ & 2,049 \\
\hline & 5 & 1,205 & 0,3477 & 0,105 & $-0,109$ & 2,519 \\
\hline & 6 & $1,9650 *$ & 0,3477 & 0 & 0,651 & 3,279 \\
\hline & 7 & 1,0975 & 0,3477 & 0,195 & $-0,216$ & 2,411 \\
\hline & 8 & $2,0450 *$ & 0,3477 & 0 & 0,731 & 3,359 \\
\hline & 1 & 0,135 & 0,3477 & 1 & $-1,179$ & 1,449 \\
\hline \multirow{6}{*}{2} & 3 & 0,325 & 0,3477 & 0,997 & $-0,989$ & 1,639 \\
\hline & 4 & 0,87 & 0,3477 & 0,511 & $-0,444$ & 2,184 \\
\hline & 5 & $1,3400 *$ & 0,3477 & 0,041 & 0,026 & 2,654 \\
\hline & 6 & $2,1000^{*}$ & 0,3477 & 0 & 0,786 & 3,414 \\
\hline & 7 & 1,2325 & 0,3477 & 0,088 & $-0,081$ & 2,546 \\
\hline & 8 & $2,1800^{*}$ & 0,3477 & 0 & 0,866 & 3,494 \\
\hline \multirow{7}{*}{3} & 1 & $-0,19$ & 0,3477 & 1 & $-1,504$ & 1,124 \\
\hline & 2 & $-0,325$ & 0,3477 & 0,997 & $-1,639$ & 0,989 \\
\hline & 4 & 0,545 & 0,3477 & 0,93 & $-0,769$ & 1,859 \\
\hline & 5 & 1,015 & 0,3477 & 0,293 & $-0,299$ & 2,329 \\
\hline & 6 & $1,7750 *$ & 0,3477 & 0,001 & 0,461 & 3,089 \\
\hline & 7 & 0,9075 & 0,3477 & 0,451 & $-0,406$ & 2,221 \\
\hline & 8 & $1,8550 *$ & 0,3477 & 0 & 0,541 & 3,169 \\
\hline \multirow{7}{*}{4} & 1 & $-0,735$ & 0,3477 & 0,724 & $-2,049$ & 0,579 \\
\hline & 2 & $-0,87$ & 0,3477 & 0,511 & $-2,184$ & 0,444 \\
\hline & 3 & $-0,545$ & 0,3477 & 0,93 & $-1,859$ & 0,769 \\
\hline & 5 & 0,47 & 0,3477 & 0,968 & $-0,844$ & 1,784 \\
\hline & 6 & 1,23 & 0,3477 & 0,089 & $-0,084$ & 2,544 \\
\hline & 7 & 0,3625 & 0,3477 & 0,993 & $-0,951$ & 1,676 \\
\hline & 8 & 1,31 & 0,3477 & 0,051 & $-0,004$ & 2,624 \\
\hline
\end{tabular}




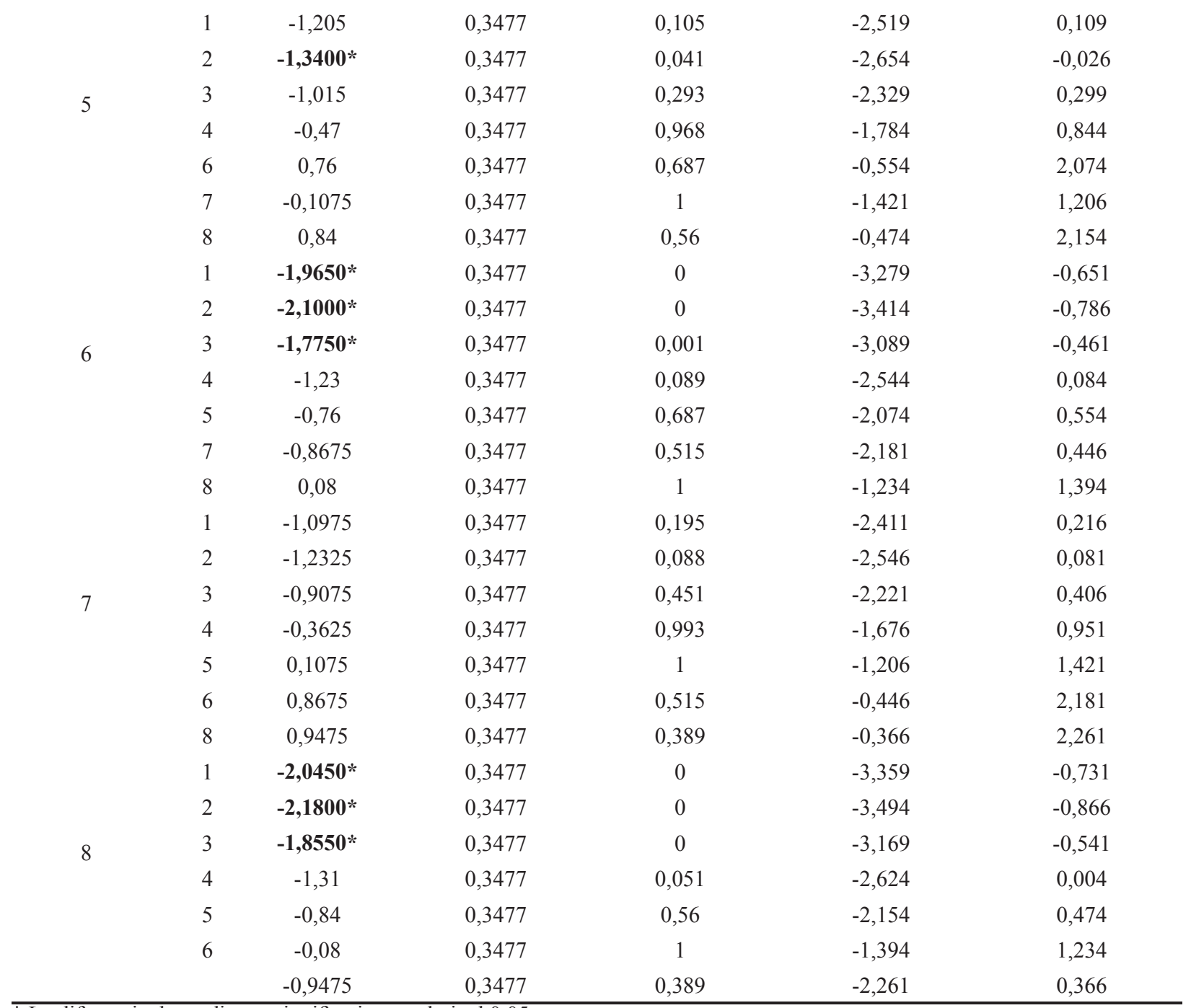

* La diferencia de medias es significativa en el nivel 0.05 .

De acuerdo con la prueba Scheffe, se evidencia que en el nivel 1, No hay diferencias estadísticamente significativas entre los puntajes del 1 al 5 ni el 7 y solo se diferencia con los puntajes de los niveles 6 y 8 .

En el nivel 2 solo se diferencia con los niveles 5, 7 y 8; en el nivel 3 solo se diferencia con los niveles 6 y 8 ; en el nivel 4 no se diferencia con ningún nivel; en el nivel 5 se diferencia con el nivel 2; en el nivel 6 solo se diferencia con los niveles 1,2 y 3 ; en el nivel 7 no se diferencia con ningún nivel y en el nivel 8 solo se diferencia con los niveles 1,2 y 3 .

\section{Discusión de Resultados}

Contribuir al mejoramiento del aprendizaje de los conceptos fundamentales de las matemáticas, en este caso las fracciones equivalentes, mixtas y reducción de fracciones mediante la innovación pedagógica es una labor de los docentes que imparten está área de conocimiento, con el propósito de obtener los mejores resultados y motivar a los estudiantes, por tanto es relevante conocer estrategias y herramientas que acompañen el aprendizaje de las matemáticas.

En la presente investigación se logró identificar que el simulador Phet aplicable a las fracciones equivalentes, mixtas y reducción de fracciones y dadas las características de su uso en línea o descargar al computador para su uso; ofrece una simulación gratuita e interactiva.

El simulador aplicado en la presente investigación se encuentra en el área de simulaciones de Matemáticas, simuladores de fraccionarios, del sitio web https:// phet.colorado.edu/es/simulation/fraction-matcher. Los cambios que se evidencian en la actualidad, abarcan prácticamente todas las actividades humanas, 
desde lo político, económico, social y ciertamente la educación. Este contexto variable interviene en la forma y estilo de vida de los individuos que conforman la sociedad, y de manera especial a los jóvenes y adolescentes.

Uno de las alternativas que existen en la actualidad es la utilización de la tecnología que ofrece una gama de herramientas para disímiles actividades de la vida diaria. Las cuales facilitan una serie de medios que se pueden valer para el trabajo docente y como estrategia de enseñanza- aprendizaje para el estudiante.

El presente estudio estableció la incidencia del uso del simulador Phet en el aprendizaje de las fracciones equivalentes, mixtas y reducción de fracciones de los estudiantes de octavo grado de Bachillerato en matemáticas de la Institución Educativa General Santander del municipio de Soacha-Cundinamarca. Arbeláez (2010) menciona que la utilización de simulación $\mathrm{y}$ de videojuegos se ha transformado en los últimos años en una poderosa herramienta de formación, lo complementa Aguirre (2012) cuando explica que la simulación se ha generalizado en los últimos 20 años en la formación de los estudiantes del mundo, debido a la necesidad que tienen los docentes de utilizar esta herramienta didáctica.

En esta investigación se trabajó específicamente en el simulador Phet en lenguaje Java aplicables a las fracciones equivalentes, mixtas y reducción de fracciones. De los resultados de esta investigación se puede aseverar que el uso del simulador Java incide en el aprendizaje de las fraccionarios se puede observar la diferencia entre los datos estadísticos del grupo de estudio, como resultado de la incidencia en uso de simuladores en el desarrollo de la unidad didáctica.

La nota promedio fue 9,575 para el primer nivel, de 9,710 para el segundo nivel, de 9,385 para el tercer nivel, 8,840 para el cuarto nivel, 8,370 para el quinto nivel, 7,610 para el sexto nivel, 8,478 para el séptimo nivel, 7,530 para el octavo nivel.

Arias (2009) resalta que la simulación puede utilizarse como un recurso para la formación de nuevos conceptos, como para la adquisición de nuevos conocimientos, destrezas, estrategias y comportamiento. Otro estudio realizado por (Méndez, 2014, p.58) donde afirma que "el uso de simuladores Java mejora el aprendizaje del teorema del trabajo-energía entre el pre-test y pos-test de los estudiantes de cuarto bachillerato".

(Pérez,2011,p.5) subraya que "Los simuladores en la educación son una herramienta muy útil de aprendizaje. Facilitan al alumno y profesor el desarrollo del conocimiento con alto grado de autonomía, comprensión de situaciones reales."

El aplicativo Phet como herramienta virtual de aprendizaje, favoreció una nueva forma de aprendizaje para estudiantes de grado octavo de la I.E. General Santander de Soacha-Cundinamarca.

Como recomendación queda accesible la eventualidad de continuar con la exploración de los que ofrece la plataforma Phet de la Universidad de Colorado, relacionado con el tema de las fracciones, analizar y evaluar el comportamiento de cada estudiante en otros grados de escolaridad con el uso del simulador y poderlo integrar al contexto escolar de la I.E. General Santander de Soacha-Cundinamarca por ser considerada esta plataforma un recurso pedagógico puesto a disposición de los docentes de matemáticas y que se pueda adaptar y formalizar en el aula de clase.

\section{Conclusión}

La metodología apoyada en la realización de trabajos de investigación con el uso de simuladores fomenta la evolución de las afirmaciones científicas del estudiante hacia un bosquejo más colindante al pensamiento científico.

A partir del análisis estadístico de los datos aplicando la prueba Scheffé, se puede observar que hay evidencia para rechazar la hipótesis nula, por tanto las medias son significativamente diferentes.

Al implementar un Objeto Virtual de Aprendizaje en la enseñanza de las matemáticas por medio de simulación Phet se evidenció una motivación por parte de los estudiantes que de acuerdo con los antecedentes que se tenían no habían tenido la oportunidad de interactuar con la tecnología dentro de una aula de una manera más dinámica en asignaturas diferentes a tecnología e informática y al aplicar este concepto a las clases de matemáticas, se evidenció en los resultados obtenidos, por el grupo de estudio una vez finalizada la fase de aplicación del O.V.A. 
Es importante mencionar la importancia que tiene el talente del Objeto Virtual de Aprendizaje y la forma en la que está elaborado ya que si no es interesante no será impactante para los estudiantes que son cada vez más visuales en el ámbito educativo.

Las Tecnologías de Información y Comunicaciones (TIC) favorecen una nueva forma de enseñanza aprendizaje, en donde se generan espacios virtuales que facilitan interacciones entre docentes y estudiantes para mejorar y desarrollar los procesos educativos en cualquier ámbito.

La aplicación del simulador Phet permitió un aprendizaje activo, participativo, característico, acrecentando el nivel de participación del estudiante, cumplimiento de tareas y actividades, y el interés por el tema de estudio, lo cual quedó demostrado.

Los estudiantes de octavo grado sostienen mayoritariamente opiniones propicias a la realización de actividades de investigación con ayuda de simuladores.

\section{BIBLIOGRAFÍA}

- Aguirre, G. (2012). Postura experiencial de los docentes que utilizan la simulación clínica como estrategia didáctica en la carrera de medicina (Tesis de Magister). Recuperado de http://www.b digital. unal. edu.co /7716/1/ 4868234.20 12.pdf

- Arbeláez, M. (2010). Mundos virtuales para la educación en salud simulación y aprendizaje en Open Simulator (Tesis de Magister). Recuperada de http://www.maestriaendiseno.com/ pdf/mauricioarbelaez.pdf.

- Arias, L. (2009). La simulación computarizada en el proceso de enseñanza aprendizaje de electrónica. Argentina: El Cid Editor.

- Bagur, A. (2011). Matemáticas para todos. Recuperado el 16 de abril de 2016, de http://www.acmor. org.mx/descargas/mate106.pdf

- Balestrini, M. (2006). Cómo se Elabora el Proyecto de Investigación. Venezuela: Servicio Editorial.

- Duran, E. (2012). Red de tecnología Educativa. Recuperado de http://reddetecnologiaeducativa.bligoo. com.co/aprendiendo-matematicas-con-la-ayuda-de-simuladores

- Fatela, M.(2012).Simuladores. Recuperado el 16 de abril de 2016, de http://www.fatela.com.ar/PaginasWeb/simuladores.htm

- García, D. (2012). Promover en el aula estrategias de aprendizaje para elevar el nivel escolar de los alumnos de tercero primaria en el área de matemáticas (Tesis de Licenciatura). Recuperada de: http:// biblio3. url.edu.gt/Tesis/2012/05/24/Garcia-Daniela.pdf

- Huapaya, G. (2012). Modelación usando función cuadrática:experimentos de enseñanza con estudiantes de 5 to de secundaria. Lima-Perù.

- Loeblin, P. (2016). Plan de lecciones para juego de fracción. Recuperado el 16 de septiembre de: https://phet.colorado.edu/es_PE/contributions/view/3571

- McGarry (2015). Fraction Matcher. Recuperado el 16 de septiembre de: https://phet.colorado.edu/services/download-servlet?filename $=\% 2$ Fteachers-guide $\% 2$ Ffraction-matcher-html-guide.pdf

- Márquez. H (2016). Software Educativo. Recuperado de: http://www.sepbcs.gob.mx/sepanmas/Cursos_Linea/Curso_Clic/edusoft.htm

- Monje, C. (2011).Metodología de la Investigación Cuantitativa y Cualitativa-Guía Didáctica. Recuperado de https://carmonje.wikispaces.com/file/view/Monje+Carlos+Arturo+-+Gu\%C3\%ADa+did\%C3 $\% \mathrm{~A} 1$ ctica+Metodolog\%C3\%ADa+de+la+investigaci\%C3\%B3n.pdf

- Muñoz, F. (2012). Simulaciones PhET para aprender Ciencias. Recuperado el 16 de Abril de 2016 de: http://recursostic.educacion.es/buenaspracticas20/web/es/difundiendo-buenas-practicas/602-simulaciones-phet-para-aprender-ciencias 
- Ortega, M. (2001). Sistemas de ineteracción persona-computador. Castilla-La Mancha: Ediciones de la Universidad Castilla-La mancha.

- Pósito, R. (2012). El problema de enseñar y aprender ciencias naturales en los nuevos ambientes educativos (Tesis de Magister). Recuperada de http://sedici.unlp.edu.ar/bitstream/handle/10915/18190/ Documento_completo.pdf? sequence $=3$

- Pérez, C. (2011). Fisim: simulador físico -matemático integrado a la plataforma de gestión del aprendizaje zera.

- Peña, P. \& Alemán, A. (2013). Teoría de simuladores. Recuperado de http://www.aves.edu.co/ovaunicor/recursos/1/index_Simulacion_por_computador.pdf

- Shuttleworth, M (2008). Diseño de la Investigación Cuantitativa. Feb 25, 2016 Recuperado de Explorable.com: https://explorable.com/es/diseno-de-la-investigacion-cuantitativa 